\title{
Quantum Cosmology and the Arrow of Time
}

\author{
Claus Kiefer \\ Institut für Theoretische Physik, Universität zu Köln, Zülpicher Str. 77, 50937 Köln, Germany \\ Received on 10 January, 2005
}

\begin{abstract}
Although most fundamental laws are invariant under time reversal, experience exhibits the presence of irreversible phenomena - the arrows of time. Their origin lies in cosmology, and I argue that only quantum cosmology can provide the appropriate formal framework. After briefly reviewing the formalism, I discuss how a simple and natural boundary condition can lead to the observed arrows of time. This yields at the same time interesting consequences for black holes.
\end{abstract}

\section{INTRODUCTION}

Recent experiments support the idea that quantum theory is universally valid. No breakdown of the superposition principle has been detected, and the disappearance of interference term can be understood in a quantitative way by the process of decoherence [1]: Entanglement with environmental degrees of freedom produces locally classical behaviour. The local system is then consistently described only by a master equation, not a unitary Schrödinger equation. Apart from microscopic and some mesoscopic systems, it is usually not possible to isolate a system from its environment. Following this chain, the environment is coupled to its environment, and so on, leading ultimately to the whole Universe as the only strictly closed quantum system. Universality of quantum theory thus dictates that the Universe as a whole has to be described by quantum theory - this is the realm of quantum cosmology. For its interpretation, no reference to an external measurement agency can be made. Since such an interpretational scheme provides insight into quantum theory in general, it was claimed that "quantum mechanics is best and most fundamentally understood in the framework of quantum cosmology" [2].

The idea of quantum cosmology is more general than the quantization of a particular interaction. However, since gravity dominates on large scales, any reasonable formalism of quantum cosmology must employ a quantum theory of gravity. Such a theory is not yet available in a definite form, but various promising approaches exist [3]. The main approaches are:

- Superstring theory (M-theory): This is a unified quantum theory of all interaction, from which quantum gravity emerges in an appropriate limit.

- Quantum general relativity: This is the application of established quantization rules to general relativity. It may lead to a viable theory on the non-perturbative level or, at least, to an effective theory away from the Planck scale. From a methodological point of view, one can further subdivide this approach: one example is the path-integral approach (Euclidean or Lorentzian), another example is canonical quantum gravity. Depending on the chosen variables, one can distinguish in the latter between, for example, quantum geometrodynamics ('Wheeler-DeWitt equation') and loop quantum gravity.

Other, even more ambitious approaches, start with fundamen- tal discrete structures such as causal sets [4]. For the present discussion it is sufficient to restrict to canonical quantum gravity, since it contains all conceptual tools that are required.

The topic addressed here is the observed irreversibility of the world and its possible justification from quantum cosmology [5]. I do not consider here the possibility of a new, fundamental, irreversible law as discussed, for example, in [6] where a fundamental master equation arises via the cosmological constant. Instead, I shall argue that the formal structure of the equations of canonical quantum gravity by themselves suggests a simple boundary condition from where the arrows of time follow naturally. I shall start with a brief review of the quantum cosmological formalism and the problem of the arrows of time. I then attempt to trace the origin of these arrows to a simple boundary condition in quantum cosmology. Finally I shall briefly discuss possible consequences for black holes.

\section{THE FORMALISM OF QUANTUM COSMOLOGY}

The central equation in canonical quantum gravity is the quantum constraint equation [7]

$$
\hat{H} \Psi=0
$$

where $\hat{H}$ denotes the full Hamiltonian of gravitational and other degrees of freedom. Equation (1) is, mostly in the geometrodynamical context, called the Wheeler-DeWitt equation. Among the important properties of (1) are:

- The quantum state $\Psi$ depends only on threedimensional quantities. It is invariant under threedimensional coordinate transformations.

- No external ('non-dynamical') time parameter is present; the state $\Psi$ describes a 'stationary wave'.

- An equation of the form (1) follows from any theory that is time-reparametrization invariant on the classical level.

- In the geometrodynamical case, (1) is pointwise hyperbolic and thus defines an 'intrinsic time'. In quantum cosmological models, the intrinsic time is given by the scale factor (or the spatial volume) of the Friedmann universe. 
Consider the simple model of a (closed) Friedmann universe with scale factor $a \equiv \exp (\alpha)$ and a homogeneous massive scalar field $\phi$ with mass $m$. The corresponding WheelerDeWitt equation then reads [8]

$$
\left(G \hbar^{2} \frac{\partial^{2}}{\partial \alpha^{2}}-\hbar^{2} \frac{\partial^{2}}{\partial \phi^{2}}+m^{2} \phi^{2} e^{6 \alpha}-\frac{e^{4 \alpha}}{G}\right) \psi(\alpha, \phi)=0 .
$$

This equation should be valid at least for scales bigger than the Planck scale: Since general relativity is the established classical theory of gravity on large scales, and since we assume quantum theory to be universally valid, the direct quantization of general relativity should be valid at least as an effective theory on large scales. It may, however, break down near the Planck scale. An ad hoc modification can be made, for example, by the introduction of an appropriate 'Planck potential' in order to facilitate the normalizability of the wave function there [9]. A more fundamental approach would be to employ results from the full theory before the restriction to homogeneous models. This can be achieved in loop quantum gravity where the spectra of geometrical operators turn out to be discrete, cf. $[3,10]$ and the references therein. For cosmological models one then finds that the Wheeler-DeWitt equation (2) is replaced by a difference equation for steps characterized by $n \in \mathbb{Z}[10]$. The number $n$ is related to the eigenvalue of the operator $\hat{p}$,

$$
\hat{p}|n\rangle=\frac{1}{6} \beta l_{\mathrm{P}}^{2} n|n\rangle,
$$

where $\beta$ denotes a quantization ambiguity (the 'BarberoImmirzi parameter'), $l_{\mathrm{P}}=\sqrt{8 \pi G \hbar}$ is the (reduced) Planck length, and $\hat{p}$ is the operator corresponding to the classical quantity $p$, where $|p|=a^{2}$. In the limit $n \gg 1$, the difference equation goes over into the differential equation (2). The number $n$ can be interpreted as playing the role of 'discrete intrinsic time'.

A most important question is how to address appropriate boundary conditions. Since there is no external time, boundary conditions have to be imposed with respect to intrinsic (dynamical) degrees of freedom. For the Wheeler-DeWitt equation in quantum cosmology, the scale factor presents itself as the appropriate timelike variable. Subtleties arise for the case where the classical model describes a recollapsing universe. If one wants to represent such classical solutions in the quantum theory by wave packets, the 'returning packet' has to be present 'initially' (with respect to an 'initial condition' for $a=$ constant). Imposing this on solutions to (2), it turns out that one cannot find a narrow wave packet all along the classical trajectory - the semiclassical approximation must breakdown somewhere [11]. This breakdown is connected with the presence of a turning point in the classical theory.

What about boundary conditions in the case of loop quantum cosmology? It turns out there that, for a particular factor ordering, the state $\psi_{0}$ corresponding to $n=0$ in the difference equation drops out. The difference equation can thus be continued through the 'classical singularity' (which would be at $n=0$ ) into the regime of negative $n$. Still, the equation that would contain $\psi_{0}$ has to be fulfilled and leads to a constraint among the other $\psi_{n}$. This is interpreted as a 'dynamical initial condition' $[10,12]$. The absence of the classical singularity is also recognized by the fact that the inverse of the operator $\hat{a}=\sqrt{|\hat{p}|}$ is bounded.

Again, the case of a closed universe exhibits subtleties because it seems that a divergent behaviour of quantum states at large scale factor cannot be avoided [13]. The origin of this problem is the fact that two possible orientations of the triads are needed in the loop approach, providing the means to continue the difference equation through $n=0$ and to avoid the classical singularity.

For the Wheeler-DeWitt equation, the question of singularity avoidance can be rigorously discussed within simple models. One is the case of a null dust shell that classically collapses to form a black hole. Demanding unitarity, a corresponding quantum theory can be constructed that fully avoids the singularity - collapsing wave packets enter the Schwarzschild radius, but then re-expand to infinity, see [3,14] and the references therein. The full solution thus describes a superposition of the black-hole and white-hole situation and is entirely singularity-free.

An important issue for any approach to quantum gravity is the semiclassical approximation. For the Wheeler-DeWitt equation, this can be achieved, at least on a formal level, by a Born-Oppenheimer type of approximation scheme, with the non-gravitational degrees of freedom adiabatically following the 'slowly developing' gravitational variables [3]. While for full loop quantum gravity this may not yet be clear, the situation in loop quantum cosmology is straightforward: For $n \gg 1$, the difference equation becomes identical to the Wheeler-DeWitt equation, and the standard BornOppenheimer scheme can then be applied.

As is well known, one can recover from the WheelerDeWitt equation in the semiclassical limit a functional Schrödinger equation for the non-gravitational degrees of freedom [3]. The corresponding time parameter is defined through the slowly evolving gravitational variables, typically the expansion of the universe. An important ingredient is decoherence of relevant variables (such as the volume of the universe) by irrelevant variables (such as small density fluctuations). Otherwise one would encounter superpositions of macroscopically different universes. Decoherence 'starts' with the onset of inflation; before inflation, the universe is timeless and there is no classical evolution [15]. In fact, due to the unavoidable quantum entanglement between matter and gravity, mutual decoherence arises. This may mimic a gravityinduced collapse of the wave function as discussed, for example, in [16]. Because of decoherence, one must use a master equation instead of the Schrödinger equation, valid for the evolution into the forward direction of (semiclassical) time $t$. If this master equation holds in that direction of $t$ that corresponds to increasing scale factor $a$, it cannot be valid across a classical turning point into a recollapsing phase. This indicates that the emergence of an arrow of time for a classically recollapsing universe is subtle, see Section IV. 


\section{ARROWS OF TIME}

Most of the fundamental laws of Nature do not distinguish between past and future, but there are many classes of phenomena that exhibit an arrow of time [5]. This means that their time-reversed version is, under ordinary conditions, never observed. Arthur Eddington called these classes 'arrows of time'. The main arrows are the following:

- Radiation arrow (one observes retarded solutions of the wave equation, but no advanced solutions);

- Thermodynamical arrow (Second Law of thermodynamics, demanding the entropy to be non-decreasing for a closed system);

- Quantum mechanical arrow (measurement process and emergence of classical properties by decoherence);

- Gravitational arrow (expansion of the universe and emergence of structure by gravitational condensation).

Since the expansion of the universe is a single process (not a class of phenomena), it has been suggested (starting from the work of Ludwig Botzmann) that it provides the root of all these arrows, the 'master arrow'. The various arguments that lead to this suggestion are discussed in great detail in [5]. It is important in this context to remind oneself that, because gravitational systems possess negative heat capacity, homogeneous states are characterized by a low gravitational entropy, whereas inhomogenous states have a high gravitational entropy. This is just the opposite than for non-gravitational systems. The maximal entropy would be reached for our universe if all matter had collapsed into a single gigantic black hole. We are obviously far away from such a state. Our universe is thus characterized by an extremely unprobable initial condition of low gravitational entropy, or, in other words: Why did the universe start so smoothly?

One can try to answer this question within the classical theory. Recent attempts include the suggestion that 'eternal inflation' may be responsible [17]. However, any fundamental attempt of explanation should address this issue in the framework of quantum gravity which transcends the classical theory. The immediate challenge to face is then the 'timelessness' of quantum gravity, cf. (1). How can one derive an arrow of time from a framework that does contain no time? I shall discuss in the following section how this can be achieved, at least in principle.

\section{ORIGIN OF IRREVERSIBILITY FROM QUANTUM COSMOLOGY}

Quantum gravity does not contain an external time parameter at the most fundamental level. As discussed above, however, one can introduce the concept of an intrinsic time, given in quantum cosmology by the scale factor $a \equiv \exp (\alpha)$ of the universe (or its discretized version). The important observation is now that the fundamental equation is asymmetric with respect to $a$. Considering a Friedmann model with small perturbations (symbolically denoted by $\left\{x_{i}\right\}$ ), the WheelerDeWitt equation (1) is of the form

$$
\hat{H} \Psi=\left(\frac{\partial^{2}}{\partial \alpha^{2}}+\sum_{i}\left[-\frac{\partial^{2}}{\partial x_{i}^{2}}+V_{i}\left(\alpha, x_{i}\right)\right]\right) \Psi=0 .
$$

The potential appearing in (4) is asymmetric with respect to 'intrinsic time' $\alpha$; one has, in particular, the important property that $V_{i} \rightarrow 0$ for $\alpha \rightarrow-\infty$. This allows one to impose a very simple boundary condition in this limit. As Zeh has suggested [5], one can demand that

$$
\Psi^{\alpha \rightarrow-\infty} \longrightarrow(\alpha) \prod_{i} \psi_{i}\left(x_{i}\right)
$$

that is, an initial condition where the various degrees of freedom are not entangled. Solving then the Wheeler-DeWitt equation with this condition, entanglement automatically increases with increasing $\alpha$. This, then, leads to an increase of the entropy for the relevant degrees of freedom which include the scale factor and some additional relevant variables $\left\{y_{i}\right\}$. The entropy is found from

$$
S\left(\alpha,\left\{y_{i}\right\}\right)=-k_{\mathrm{B}} \operatorname{tr}(\rho \ln \rho)
$$

where $\rho$ is the reduced density matrix obtained by integrating out all irrelevant degrees of freedom from the full quantum state. This increase of entropy then defines the direction of time. All the arrows of time discussed in Section III would then have their common root in this entropy increase. The emergence of a correlated state from the symmetric initial state (5) then represents a 'spontaneous symmetry breaking' similar to the symmetry breaking when the quantum field theoretic vacuum proceeds from a symmetric to an asymmetric state [5], cf. also [9]. Since the time parameter $t$ in the semiclassical limit is defined as a function of the scale factor, time is defined by the expansion of the universe. In a sense, the expansion of the universe is a tautology. It would be interesting to perform the above analysis for the difference equation of loop quantum cosmology.

What happens for a universe that is classically recollapsing? Since the boundary condition (5) is formulated for $\alpha \rightarrow-\infty$, irrespective of any classical trajectory, it applies at the same time to the 'big bang' and the 'big crunch'. Only one condition is thus needed in order to cover both regions. Consequently, increase of entropy is always correlated with increase of scale factor, that is, increasing size of the universe. But what happens at the turning point? There the arrow of time reverses, but the reversal is only of formal significance. Since the semiclassical approximation breaks down there [11], the universe is fully quantum in this region - no classical observer could survive it [18]. Many quasi-classical components of the full quantum state, each representing a universe of its own, interfere there destructively in order to fulfill the final condition of the wave function going to zero for $\alpha \rightarrow \infty$. Quantum cosmology thus not only specifies the beginning of the classical evolution (when decoherence sets in at the onset of inflation), but also its end. 


\section{CONSEQUENCES FOR BLACK HOLES}

A fundamental quantum cosmological boundary condition such as (5) has also profound consequences for black holes in a recollapsing universe $[5,18]$. Consider an object, for example a dust shell or a star, that collapses to form a black hole (assume a Schwarzschild black hole, for simplicity). The collapse is supposed to happen (in the proper time of the collapsing object) long before the universe as a whole reaches its maximum expansion at, say, a Schwarzschild time $t_{\text {turn }}$. Since the full quantum cosmological boundary condition is symmetric, the collapsing object must expand again for $t>t_{\text {turn }}$, although any observer would experience this as collapsing (because his arrow of time always points from small $a$ to large a). Because of the mentioned quantum effects near the turning point, no classical connection exists between the collapse of the object and its following (formal) expansion. As a consequence, one has [18]

- no horizon formation,

- no singularity inside the black hole, and therefore

- no information-loss problem and

- no need to introduce cosmic censorship, since also no naked singularities form.

Unfortunately, these consequences cannot be tested from outside (due to the large redshift), but only by volunteers plunging into the black hole - they would enter the quantum era of the cosmological turn-around within a short proper time.

Recently, an ad hoc final state boundary condition was imposed at black-hole singularities in order to prevent information from being absorbed by the singularity [19]. Like in the case discussed here, the corresponding quantum state consists of a superposition of many macroscopically distinct states. However, in our case this consequence follows directly from the fundamental framework of quantum cosmology - and the 'information-loss problem' does not exist because neither a horizon nor a singularity would ever form. A final answer can, of course, only be obtained after the full theory of quantum gravity has been constructed and experimentally tested.

\section{ACKNOWLEDGMENTS}

I would like to thank the organizers of DICE2004, and in particular Hans-Thomas Elze, for inviting me to this wonderful and inspiring meeting.
[1] E. Joos, H.D. Zeh, C. Kiefer, D. Giulini, J. Kupsch, and I.O. Stamatescu, Decoherence and the Appearance of a Classical World in Quantum Theory, second edition (Springer, Berlin, 2003). See also: www.decoherence.de

[2] M. Gell-Mann and J.B. Hartle, in: Complexity, Entropy, and the Physics of Information, edited by W.H. Zurek (AddisonWesley, Reading, 1990).

[3] C. Kiefer, Quantum Gravity (Clarendon Press, Oxford, 2004).

[4] R. Sorkin, gr-qc/0309009 , see also his contribution to these Proceedings.

[5] H.D. Zeh, The physical basis of the direction of time, fourth edition (Springer, Berlin, 2001). See also: www.time-direction.de .

[6] N.E. Mavromatos, gr-qc/0411067 , contribution to these Proceedings.

[7] This is the form for a closed universe. In the open case (relevant, for example, for black holes), boundary terms are present [3].

[8] Units are chosen such that $c=1$ and $G \rightarrow(3 \pi / 2) G$.

[9] H.D. Conradi and H. D. Zeh, Phys. Lett. A 154, 321 (1991).
[10] M. Bojowald and H.A. Morales-Técotl, in: The early universe and observational cosmology, edited by N. Bretón, J.-L. Cervantes-Cota, and M. Salgado (Springer, Berlin, 2004).

[11] C. Kiefer, Phys. Rev. D 38, 1761 (1988).

[12] M. Bojowald, Phys. Rev. Lett. 87, 121301 (2001).

[13] D. Green and W.G. Unruh, Phys. Rev. D 70, 103502 (2004).

[14] P. Hájiček, in: Quantum gravity: from theory to experimental search, edited by D. Giulini, C. Kiefer and C. Lämmerzahl (Springer, Berlin, 2003).

[15] A.O. Barvinsky, A.O. Kamenshchik, C. Kiefer and I.V. Mishakov, Nucl. Phys. B 551, 374 (1999).

[16] L. Diósi, quant-ph/0412154 , contribution to these Proceedings.

[17] S.M. Carroll and J. Chen, hep-th/0410270 ; H. Nikolić, hep-th/0411115.

[18] C. Kiefer and H.D. Zeh, Phys. Rev. D 51, 4145 (1995).

[19] G.T. Horowitz and J. Maldacena, JHEP 0402, 008 (2004). 